\title{
Modernidad y Ciencias Sociales: Una propuesta epistemológica interdisciplinaria en la Teoría Crítica
}

\author{
Modernity and Social Sciences: An interdisciplinary epistemological proposal in critical theory \\ Francisco Omar Peña Guajardo ${ }^{a}$
}

\begin{abstract}
:
This research article analyzes the epistemology of the Frankfurt School's critical project, proposes a new approach to orthodox Marxism and suggests the emergence of an interdisciplinary project that questions the foundations of an epistemological traditionalism in the face of the modern crisis. It is worth mentioning that there is a tour of the three generations of the critical project, to understand its complexity and finally, to establish the bases for the construction of a critical epistemology and capable of giving an emancipatory explanation to the different social realities that take place in the modern world.
\end{abstract}

\section{Keywords:}

Modernity, Social Sciences, Critical Theory, Epistemology, Interdisciplinarity

\section{Resumen:}

El presente artículo de investigación, realiza un análisis sobre la epistemología del proyecto crítico de la Escuela de Frankfurt, tal propuesta, replantea al marxismo ortodoxo y sugiere la emergencia de un proyecto interdisciplinario, que cuestiona los fundamentos de un tradicionalismo epistemológico ante la crisis moderna, cabe mencionar, que se hace un recorrido de las tres generaciones del proyecto crítico, para comprender su complejidad y finalmente, establecer las bases para la construcción de una epistemología crítica y capaz de dar una explicación emancipadora ante las diferentes realidades sociales, que acontecen en el mundo moderno.

\section{Palabras Clave:}

Modernidad, Ciencias Sociales, Teoría Crítica, Epistemología, Interdisciplinariedad

\section{INTRODUCCIÓN}

El presente artículo tiene como propósito, dilucidar los diferentes alcances que tiene la epistemología moderna, entendiéndose como aquella integrada a un discurso de legitimidad y validez, relacionada principalmente a la filosofía kantiana y hegeliana. Por otra parte, la construcción de las Ciencias Sociales, originó sus bases en la filosofía positivista, teniendo por resultado que dichas ciencias se institucionalicen.

La crisis del proyecto moderno puso en discusión la validez del conocimiento científico, originando posturas opuestas, entre ellas surge la Teoría Crítica como una manera de pensar y hacer ciencia, reestructurando la epistemología clásica o tradicional.

La Teoría Crítica*, llamada así a la Escuela de Frankfurt, se consolida posterior a la segunda guerra mundial,

\footnotetext{
* Es importante enfatizar que el proyecto de la Teoría Crítica, es desarrollada en el siglo XVIII y XIX con los denominados hegelianos de
}

estableciéndose como una manera de pensar y hacer filosofía y ciencia social a la vez, pues en ella se encuentra un modelo enriquecedor, donde se conjuntan distintas perspectivas teóricas, tales como: el marxismo, el psicoanálisis, la psicología social, el estructural funcionalismo, el interaccionismo simbólico, la fenomenología, la sociología comprensiva y la hermenéutica, dando lugar a una teoría interdisciplinaria para el análisis de las diferentes realidades históricas y sociales en las sociedades occidentales $\mathrm{y} / \mathrm{o}$ occidentalizadas.

Para cumplir con el objetivo del trabajo, es necesario señalar a las diferentes generaciones en las que surge el proyecto crítico, refiriendo a la primera, segunda y tercera izquierda: Ludwing Feuerbach, Arnaldo Ruge, Max Stirner, Friedrich Engels y Karl Marx, como se señalará en el transcurso del trabajo. 
generación*. También se realizará un recorrido teórico, en el que se indicarán sus particularidades y preocupaciones como un proyecto en oposición al convencionalismo científico, generando un programa de investigación social, que tiene por objetivo desarticular a la ortodoxia científica moderna y que posibilita otros modos en que se puede analizar los distintos fenómenos sociales de la vida social moderna.

\section{Modernidad y Ciencias Sociales}

El proyecto de la modernidad se establece a partir del siglo XVI en Europa y posteriormente se desplaza a otros territorios, principalmente a África, Asia y América Latina El orden moderno se desglosa en distintos procesos históricos tales como: el renacimiento italiano, la reforma protestante luterana, la ilustración francesa y el parlamento inglés. El nacimiento de la modernidad según Enrique Dussel tiene una fecha en específico: "[...] la modernidad comienza en 1492 con el descubrimiento de América, teniendo a España como el país por antonomasia en donde se da la apertura de Europa, siendo el atlántico como el centro geopolítico de la modernidad" (Dussel, 2015, s.p.).

Es importante tomar en cuenta que Europa se está abriendo al mundo, reinventando y rompiendo los viejos cánones a los que por siglos estuvo sujeta, tal como el eclesiástico. La ruptura con el orden eclesiástico, trajo consigo la articulación de un nuevo tipo de discurso basado en la razón; una razón de tipo científica. El nacimiento de la ciencia viene a constituir el nuevo tipo de discurso o conocimiento legítimo y válido en la modernidad.

La ciencia es el proyecto de la modernidad y la modernidad el proyecto del ser occidental, particularmente el que se emancipa y posiciona con una capacidad de raciocinio, capaz de ejercer un juicio propio o una crítica a su contexto histórico, social, cultural y político.

En el siglo XVIII en Europa, el pensamiento de Immanuel Kant (1724-1809), tendría una influencia en la física newtoniana, relacionada a la concepción mecanicista, en su obra, Crítica de la razón pura (1781), plantea como la física y la matemática son ciencias por ser verificables, la primera por tener una forma apriorística en la sensibilidad externa, es decir; en el mundo material y espacial. La segunda postura según el autor, se manifiesta de manera interna, concibiendo el tiempo y/o la temporalidad humana. De tal forma que estos dos elementos: espacio y tiempo, conforman en conjunto el mundo de lo fenoménico, sentando las bases de la epistemología moderna.

\footnotetext{
* La primera generación está representada por Max Horkheimer y Theodor Adorno, la segunda generación por Jürguen Habermas y la tercera y última generación por Axel Honneth.

${ }^{\dagger}$ Es importante señalar como a partir de este momento las Ciencias Sociales construyen una dicotomía: Nomotéticas e Ideográficas, la primera alude al
}

La metafísica es otro tipo de conocimiento diferente al de la ciencia, al no formar parte del mundo fenoménico y estar relacionada con la moral. La metafísica se desconoce como conocimiento de validez por no ser comprobable. Por ello Immanuel Kant viene a hacer la síntesis del conflicto generado siglos atrás, entre racionalistas y empiristas. Los primeros representados por René Descartes, quienes concebían al conocimiento como un elemento que se genera de forma innata en la conciencia del individuo; mientras que los segundos, los empiristas, David Hume, sería quien se destaca de esta escuela, retoma la experiencia como el origen de donde surge el conocimiento. Por lo que, ambos enfoques se van a considerar como pilares, para el surgimiento de las Ciencias Sociales.

Las Ciencias Sociales surgen como una necesidad humana y de la época; la ruptura con el viejo orden religioso y las ideas de la ilustración permearon la vida económica, política, social y cultural del viejo y a la vez del nuevo occidente que se estaba formando. Los procesos de industrialización, la ascensión del capitalismo como modelo hegemónico y la ruptura con los viejos órdenes políticos, principalmente el monárquico y la ascensión de la burguesía, se establecen como la nueva lógica en la que se rige el proyecto de la modernidad.

Tales ciencias retoman e intentan reproducir la forma de conocimiento bajo el cual están elaboradas, las ciencias naturales a las que llaman positivismo, siendo Auguste Comte uno de los precursores de dicha escuela filosófica. Comte también pionero de la Sociología, propone la reintegración social de la sociedad francesa a través del conocimiento científico. Para él, la historia ha transcurrido por diferentes etapas o estadios: el religioso, metafísico y científico, el último corresponde a la época que estaba aconteciendo.

El nacimiento del positivismo, da apertura a que se institucionalicen las Ciencias Sociales, la Sociología, Ciencia Política y Economía. Por otro lado, la Historia y Antropología más que tomar un enfoque cientificista de corte positivista, sus estudios se crean para comprender e interpretar los fenómenos históricos y culturales, dando pauta a un debate epistémico en las Ciencias Sociales y considerando el enfoque más loable para el análisis de las realidades sociales ${ }^{\dagger}$. Sus estudios se centraron principalmente en los países de Gran Bretaña, Francia, Alemania, Italia y Estados Unidos, también hubo otros que se centraron en comparar las sociedades orientales con las occidentales, un ejemplo de ello son los estudios que realizó Max Weber en su obra: Economía y Sociedad (Wallerstein en Salgado, 2002).

Las Ciencias Sociales crecen a la par del desarrollo de la modernidad, por eso es importante señalar que la crisis

establecimiento de leyes, partiendo de un método hipotético deductivo, entre estas se encuentran: la Economía, Ciencia Política y Sociología, y la segunda, aboga por la comprensión y la subjetividad, teniendo un método inductivo como: la Historia y Antropología. 
de la modernidad deviene de una crisis científica, propuesta que se empezó a plantear en Europa durante el siglo XX, construyéndose así un enfoque de corte crítico al que nos referiremos a continuación.

En el siglo XVIII cuando se da la filosofía de I. Kant, se establece una separación y una contraposición entre lo a priori y a posteriori, el fenómeno y el noúmeno, este último relacionado con lo incognoscible, a lo que llamó el autor "cosa en si"". Sin embargo, en el siglo XIX con el idealismo metafísico de G. W. F. Hegel y su propuesta dialéctica, termina espiritualizando y diluyendo las contradicciones inherentes entre esta bifurcación:

[...] Hegel en cuanto pone
al espíritu absoluto como
eminentemente real (das
Allerrealste). Lo universal,
según él, ya se ha
desplegado
adecuadamente y es
idéntico a lo que se
concreta. La razón ya no
necesita ser simplemente
crítica respecto de sí
misma; en Hegel ella se ha
vuelto afirmativa; aun antes
de que la realidad deba ser
afirmada como racional
(Horkheimer, 2003, p. 237).

En el siglo XIX con la creación de nuevos modelos políticos y sociales, principalmente el burgués y de filosofías como: el idealismo alemán de G. W. F. Hegel y el positivismo francés de A. Comte. La ciencia y en especifico las Ciencias Sociales se constituyen como saberes científicos. Es importante señalar, que la crítica a la ciencia y a las Ciencias Sociales, se explícita de manera más clara en el Siglo XX, sin embargo, en el XIX la construcción de una Teoría Crítica estaba en proceso.

\section{Epistemología y Teoría Crítica}

La Teoría Crítica surge en Alemania en 1840 entre los jóvenes hegelianos de izquierda, se consideran a esta época como una etapa de gran efervescencia intelectual debido al legado de Hegel. Citando a Laura Páez Díaz de León: "[...] los jóvenes hegelianos de izquierda representaron una apertura hacia la crítica social secularizada. La crítica de la religión, desde el materialismo crítico de Ludwing Feuerbach dejó atrás la exégesis dogmática, que había dominado en la Edad Media, para reafirmarse como crítica social" (Páez, 2009, p. 27).

\footnotetext{
* La cosa en sí se opone al fenómeno puesto que el fenómeno es la cosa tal como aparece, mientras que el objeto= x no se opone del todo al fenómeno, es la referencia de todo fenómeno a la forma de objeto. La cosa en sí está situada fuera de nuestro conocimiento posible, puesto que nosotros solo conocemos lo que aparece. Deleuze, Gilles, Cuatro lecciones sobre Kant dictadas entre marzo y abril de 1978. Edición electrónica de www.philosophia.cl, Escuela de Filosofía Universidad. ARCIS,

p.63.http://minhateca.com.br/rfbiblioteca/Biblioteca/DELEUZE*2c+Gilles
}

Los teóricos críticos de 1840 proponen una crítica social, concibiendo un sujeto histórico concreto en el que se desarrolla el proyecto revolucionario al que llamaron proletariado. Por otra parte, los integrantes del Institut Für Sozialforschung ${ }^{\dagger}$, encontraron más complicaciones debido al debilitamiento de la clase obrera revolucionaria a mediados del siglo XX. El enfoque del instituto tenía una postura enfocada principalmente al marxismo, sin dejar de lado, otras propuestas de otros pensadores: Schopenhauer, Nietzsche, Dilthey, Bergson, Weber, Husserl, entre otros: "Cómo resultado la Teoría Crítica tuvo que afirmarse frente a un cierto número de competidores que habían desalojado a Hegel del terreno. $Y$ por supuesto no pudo evitar ser influida por algunas de sus ideas" (Jay, 1989, p. 86).

El Institut für Sozialforschung fue una institución académica asociada a la Universidad de Frankfurt, posteriormente Ilamada Escuela de Frankfurt luego de 1933, período en que el Partido Nacional Socialista Obrero Alemán llega al poder: "[...] la noción de una escuela especifica no se desarrolló hasta que después de que el Institut se viera forzado a abandonar Frankfurt (el término no fue empleado hasta que el Institut regresó a Alemania, en 1950)" (Jay, 1989, p. 14).

El 3 de febrero de 1923 nace el Instituto de Investigación Social en Frankfurt por un decreto del Ministerio de Educación (Laurent, 1991, 4), quedando en acuerdo con la Gesellschaft fur Sozialfors e inaugurada de manera oficial: "[...] el 22 de junio de 1924" (Jay, 1989, p. 14), siendo Félix J. Weil el primer director. La propuesta es construir un marxismo teórico y político.

La figura de Max Horkheimer es importante en la conformación del instituto; junto con Weil, se interesaron en temas de antisemitismo y del movimiento obrero. Horkheimer plantea un enfoque interdisciplinario e incluye el análisis de la psicología social para superar la fragmentación individuo sociedad. Posteriormente, Erich Fromm, Karl Landauer y Herbert Marcuse se incorporan para comenzar un proyecto dialéctico en el Instituto (Jay, 1989).

Adorno, quien se integra de manera oficial en 1938, fue uno de los principales personajes junto con Horkheimer, en la consolidación del proyecto crítico: "El trabajo de la Escuela de Frankfurt estuvo apoyado en una filosofía social cuya articulación, durante la década del treinta, fue la ocupación esencial de Horkheimer, Marcuse y, en menor medida, Adorno. Fue aquí donde su reformulación del marxismo tradicional llegó a ser fundamental. Así debemos ahora considerar la génesis del desarrollo de la Teoría Crítica" (Jay, 1989, p. 82).

$1 * 23 * 23 * 23+$ Deleuze $* 2 c+$ GuillesCuatro+lecciones+sobre+Kant, 3020066 94.pdf.

† Instituto de Investigación Social, nombre con el que primero se dio a conocer la Escuela de Frankfurt, antes de que se llamara Instituto de Investigación Social, la propuesta era Instituto para el Marxismo pero este último se descarto debido al contexto antisemita. 
La propuesta epistemológica de la primera generación de la Teoría Crítica, realiza una crítica a los sistemas filosóficos cerrados, refiriéndose a la filosofía positivista y a la dialéctica hegeliana, por ser dos modelos universalistas y no tener una relación con la realidad histórica concreta. Las contradicciones entre teoría y realidad se diluyen quedando al descubierto las relaciones de poder y dominación burguesas. La crítica a la racionalidad instrumental creada en el orden capitalista moderno, sentaron las bases para recuperar lo histórico y concreto de la realidad social que se encuentra en su negación. La teoría como praxis fue el referente epistémico bajo el cual se construye la Teoría Crítica en esta primera etapa de pensamiento*.

Una de las propuestas epistemológicas de relevancia en la primera generación de la Teoría Crítica, es la de Theodor Wisenground Adorno, al publicarse en 1966 Dialéctica Negativa, obra en que desarrolla su postura epistemológica. Señalando que el trabajo con Max Horkheimer, se analiza en Dialéctica de la Ilustración Fragmentos Filosóficos escrita en 1944 y publicada en 1947 durante su exilio en Estados Unidos.

Ahora bien, la dialéctica negativa retoma el materialismo dialéctico de Karl Marx y crítica a Hegel, el cual anula las contradicciones entre espíritu y naturaleza, reduciendo lo histórico y concreto de la realidad social occidental. La propuesta teórica de Adorno es crítica por ser dinámica, encargada de visibilizar los antagonismos generados entre sujeto y objeto. La dialéctica metafísica de Hegel está compuesta en una unidad, unidad que es criticada por el análisis materialista de la dialéctica negativa, en la cual se establece una negación a la unidad. Lo no idéntico es el principio que desarticula la universalidad, que retoma un carácter histórico:

Desde el momento en que Hegel, consecuentemente, resuelve lo no-idéntico en la pura identidad, el concepto se convierte en garante de lo no-conceptual, la trascendencia es capturada por la inmanencia del espíritu y convertida en la totalidad de éste abolida. Cuanto más, por obra de la llustración, se va descomponiendo luego en el mundo y en el espíritu, tanto más se convierte la trascendencia en algo oculto, como si por encima de todas las mediaciones se concentrara en una cima máxima (Adorno, 2008, p. 368).

La filosofía de Adorno está comprometida en establecer una filosofía crítica que sea autocrítica, de la cual sus elementos teóricos y prácticos se entretejen sin dejar de ser opuestos y sin negar la opresión creada por la modernidad. "Puesto que el carácter fundamental de todo concepto universal se desvanece al ente determinado, la

\footnotetext{
* Otra de las propuestas más enriquecedoras en establecer la relación TeoríaPráctica, fue el análisis del italiano Antonio Gramsci, quien retoma el papel de la filosofía, como un quehacer basado en la crítica y en la intervención de las diferentes transformaciones sociales, en la consolidación de las Ciencias Sociales, el papel del científico social, es contribuir a que se gesten los procesos de cambio social, por ejemplo, planteado desde la perspectiva
}

filosofía no puede seguir teniendo puestas sus esperanzas en la totalidad" (Adorno, 2008: 134).

Esta dialéctica negativa, crítica al positivismo, por cubrir un sujeto pasivo y acrítico a su realidad concreta e histórica. La propuesta que tiene está encaminada en dos niveles, plantear las contradicciones inherentes en el conocimiento y en la sociedad moderna. La filosofía de Adorno es una reflexión metafísica que construye una teoría crítica: "Crítica, negación y dialéctica son ideas fundamentales en el pensamiento de Adorno y, en general, en toda la Escuela de Francforth" (Barahona, 2006, p. 204).

La irracionalidad del siglo XX fue resultado de un proceso de racionalización, en la cual el individuo como particularidad es sometido a ser tratado dentro de una universalidad. Durante la primera generación de la Escuela de Frankfurt, se realiza una crítica al concepto de racionalidad instrumental y a sus distintas manifestaciones en las que se expresa, la ciencia, el arte, el Estado y el capitalismo.

Por otra parte, Jürgen Habermas, quien fue alumno de Adorno, es una figura necesaria en el desarrollo de la Escuela de Frankfurt y para que la Teoría Crítica se consolide de otros elementos distintos al marxismo y la filosofía hegeliana, tales como: la teoría estructural funcionalista de Talcott Parsons, el interaccionismo simbólico de George Herbert Mead, la hermenéutica de Hans George Gadamer y la fenomenología de Alfred Schütz. Construye un aparato teórico y discursivo de la sociedad moderna y analiza su funcionamiento, al que llama: teoría de la acción comunicativa. Para Habermas, el proyecto de la modernidad es un proyecto inacabado que se opera en dos mecanismos de integración: la sistémica y la social (Galindo, 2003).

La integración sistémica se establece principalmente en la teoría estructural funcionalista de Talcott Parsons, retomando la idea de economía y política de este, para realizar una distinción en el modo en que se reproduce el sistema social. Para Habermas, la economía es representada por el dinero y la política por el poder. La primera, refiere que para comprar una cosa solo se necesita pagar por el precio. El segundo, es una forma estratégica que se ejerce en los individuos. Ambos elementos, dinero y poder, son coercitivos en las relaciones sociales que se construyen de manera comunicativa, a esto el autor le llama: "[...] situación ideal del habla" (Galindo, 2003, p. 64).

En cuanto a la integración social, esta se constituye en el mundo de la vida, separada de las filosofías de la conciencia $^{\dagger}$, en este mundo, se desarrolla la intersubjetividad y el espacio de la comunicación. Pero el

de esta Primera Generación y en Gramsci. Vease: Bradner, Johann (1999) Sozialarbeit und Philosophie der Praxis in "Forum Kritische Psychologie", no. 40, traducción del alemán al español: Sieglin, Veronika, Trabajo Social y Filosofía de la Praxis. p.p. 1-9.

${ }^{\dagger}$ Filosofías de la conciencia, se alude principalmente a la filosofía de Descartes al conceptualizar la idea de un "yo" insociable. 
mundo de la vida, se ha visto afectado por la racionalidad instrumental, estableciéndose así la colonización del mundo de la vida. Es necesario destacar que Habermas reconoce el consenso como una alternativa para la descolonización de este mundo, por ello retoma el imperativo kantiano y traslada la razón práctica de este plano al de la racionalidad comunicativa. En ella se reconstruye el lenguaje, medio en donde no solo se establecen las interacciones sino también las formas de vida. Cabe aclarar que esta relación: sistema y mundo de la vida, es mediado por el espacio público, constituido por el derecho, en el que se acuerdan las leyes que los gobernarán haciendo uso de su autonomía (Galindo, 2003).

La propuesta teórica de la segunda generación, sirvió para romper los límites de la negatividad en la cual se situó la primera generación, principalmente las propuestas de Horkheimer y Adorno, quienes se basaban en el materialismo dialéctico de Marx para develar las relaciones de poder y dominación que encubría el conocimiento científico moderno.

Por ello, la lectura habermasiana se puede entender como un modelo teórico discursivo y consensual no solo por su teoría sino también por la relación que establece entre filosofía y ciencias sociales, siendo su análisis interdisciplinario. Estudia la ciencia desde un enfoque comprensivo e interpretativo, desestructurando la lógica causal cientificista, atribuida a la tradición positivista. La perspectiva habermasiana, se compone de la comunicación, elemento que sirve para mediar las relaciones de dominación y colonización en la vida cotidiana, de ahí que sea de nuestro interés explicarla e integrarla al trabajo.

La teoría de Habermas tuvo una importante aportación teórica en Europa en los años 80s, siendo un referente epistemológico de las Ciencias Sociales, aunque en los últimos años, el proyecto de la Teoría Crítica se ha ido modificando y ampliando, reconstruyendo sus fronteras y analizando nuevos fenómenos que se han incorporado a la vida social moderna, como: la desigualdad social, el multiculturalismo y el reconocimiento. Es en este proceso donde se desarrolla la tercera generación de la Escuela de Frankfurt, teniendo a Axel Honneth como principal representante.

Las luchas sociales que se suscitaron en el siglo XVIII Y XIX en Europa, refiriéndome a la revolución burguesa y proletaria, rompieron el límite de los valores morales de la sociedad occidental de aquella época, fundando nuevas luchas por el reconocimiento (Tello, 2011), consecuentes de un proceso evolutivo e histórico, donde Honneth retomaría el trabajo de Hegel.

La teoría del reconocimiento de Axel Honneth, es una recuperación de los escritos en Jena de Hegel. En los que presenta las distintas formas de interacción expuestas por el filósofo alemán, como formas de reconocimiento intersubjetivo, quedando lo normativo como intereses de reconocimiento recíproco en la que se constituye una lucha social por el reconocimiento (Sampaio, 2009).
Honneth tiene una tendencia a estudiar el reconocimiento en la esfera del derecho y en la esfera de la solidaridad. La esfera del derecho tiene un enfoque universalista en todos los espacios de la sociedad, mientras que la esfera de la solidaridad es contextual y particularista en el sentido de que un sujeto se diferencia de otro.

No obstante, influenciado por el pensamiento hegeliano en cuanto a la separación que realiza de familia, Estado y sociedad civil, propone tres formas en las que se expresa el reconocimiento: "[...] la del "amor", la del "derecho" y la de la "eticidad" (Sampaio, 2009, p. 207). Estas tres formas de reconocimiento pertenecen a tres dimensiones de la personalidad del sujeto y responden a tres tipos de autorrelación práctica de este mismo.

El amor concierne a la relación afectiva, en un plano no solo del amor sino también de la amistad que crea autoconfianza en el sujeto. El derecho corresponde a lo jurídico y con respecto al sujeto, como ser autónomo, genera una responsabilidad de tipo moral llevando al autorespeto. Por último, la solidaridad, representada en una comunidad de valores compartidos, se relaciona a la valoración social que tiene el sujeto sobre sí mismo (Sampaio, 2009), entendiéndose como la autoestima. A estas formas de reconocimiento se oponen tres formas de desprecio que se manifiestan en la carencia de autoconfianza, autorespeto y autoestima.

El objetivo de Honneth es elaborar una teoría moral del sufrimiento humano, resultado de la falta de reconocimiento, por ello es que edifica su teoría en las bases del interaccionismo simbólico de George Herbert Mead, alejándose del análisis metafísico hegeliano.

El sujeto para él, se construye en relación con otros sujetos en el espacio intersubjetivo de interacción, por ello es que el reconocimiento es el elemento fundamental en el que se configura la subjetividad humana. Por otra parte, las estructuras en las que se manifiestan las formas de reconocimiento son esenciales para la constitución de la sociedad.

La teoría del reconocimiento tiene una carga filosófica, sociológica y psicológica, herencia de las tradiciones de Adorno, Habermas y Mead, también de los idealismos del XIX como Kant y Hegel. La aportación epistemológica que tiene esta teoría en el estudio de las Ciencias Sociales, posibilita pensar y construir al objeto y al sujeto de estudio como un ser dinámico que se encuentra en constante recreación con su otro, del que se diferencia y a la vez se reconoce. De manera que se amplia y enriquece el análisis de lo social, en aras de un contexto histórico social desigual y en crisis, diluyendo la solidez en la que fueron construidos los preceptos de la ciencia y en especifico de las Ciencias Sociales, con una carga atribuida principalmente a la tradición positivista.

\section{Conclusiones}


Las Ciencias Sociales tienen un origen positivista, pero hay que tener en cuenta que a mediados del siglo $X X$, hubo una reestructuración en su programa científico, que se establece del siguiente modo:

1).- La emergencia de una propuesta teórico crítica al tipo de racionalidad instrumental moderna ante el nacimiento de la Escuela de Frankfurt.

2).- La actualidad de esa Teoria Crítica que surgió a partir de la Escuela de Frankfurt.

3).- Nuevas tradiciones teóricas epistemológicas que han surgido (además de la Teoría Crítica de Frankfurt), en las Ciencias Sociales que posibiliten repensar el papel crítico e interdisciplinar de estas y su quehacer en la sociedad.

1.- La Teoría Crítica es una corriente de pensamiento que se puede entender y explicar como aquella que se contrapone a los supuestos modernos epistemológicos, en donde se recrea a un sujeto histórico del que se emanan relaciones de poder y dominación. Horkheimer y Adorno clarifican estas relaciones como consecuencia de un sistema de producción capitalista, que transforma en calidad de "cosa" al sujeto.

La instrumentalización de la ciencia, deviene para que se consolide un programa crítico, plural y ecléctico para el análisis de las diferentes realidades sociales en occidente. El proyecto crítico incluye la hermenéutica, la fenomenología, el estructural funcionalismo y el interaccionismo simbólico para construir un aparato teórico epistémico, en donde la comunicación ocupa una posición privilegiada al ser mediadora entre el sistema y el mundo de la vida cotidiana, tal como propone J. Habermas.

2.- La Teoría Crítica en la actualidad ha sido utilizada para el estudio de las minorías, los excluidos y los otros. La teoría del reconocimiento de Axel Honneth se compone de tres elementos que se configuran en la subjetividad del sujeto: el amor, el derecho y la eticidad; también establece cómo se fortalece la relación con los otros en el mundo de la intersubjetividad humana. Por otro lado, se enfoca en destacar el papel del reconocimiento en el derecho, porque es en él donde se estructuran las acciones humanas.

La cosificación, el conflicto, el poder, la dominación y lo otro, han sido las principales preocupaciones de la Teoría Crítica, sin reducir su potencial epistemológico. Esta teoría ha tenido un proceso de ruptura, trascendiendo sus límites y llevando sus discusiones fuera del círculo de Frankfurt, tal es el caso de los posestructuralistas franceses: Michel Foucault, Jaques Derrida y Gilles Deleuze, quienes reconstruyen un aparato epistémico crítico cuestionando las diferentes formas en que se reproducen los discursos legitimados por la ciencia moderna.

3.- Por otra parte, la aportación del estructuralista francés Louis Althusser, quien sería otro heredero de un pensamiento crítico marxista y en que en su obra: Los aparatos ideológicos del Estado (1970), propone que la escuela, la religión, los medios de comunicación, el trabajo y el sindicato, son elementos reproductores de la verdad, generados a partir de las creencias que el individuo va interiorizando a partir de los diferentes procesos de socialización, estas concepciones que tiene este de sí mismo, no será más que planteadas por una clase dominante y en forma de ideología se van instituyendo. Si cree en la justicia, se someterá sin discutir a las reglas del derecho, podrá incluso protestar cuando sean violadas, firmar petitorios, tomar parte en una manifestación, etcétera (Althusser, 1998).

Mientras tanto, en la Sociología Estadounidense, otra propuesta, que se propone, es en la Sociología Radical de C. Wrigth Mills, pues fue otra de las aportaciones en mantener una postura crítica desde las Ciencias Sociales.

En América Latina con la filosofía de la liberación de Enrique Dussel, fija las bases de una Teoría Crítica latinoamericana, sin negar la influencia teórica de Dussel con Walter Benjamin, personaje importante en el desarrollo de la primera generación de Frankfurt, principalmente en el pensamiento de Theodor Adorno.

En los últimos treinta años, con la aparición de la Teoría de Sistemas de Niklas Luhmann y la caída de los metarrelatos de Jean Francois Lyotard que da inicio a otro proceso llamado posmodernidad, pese a las críticas que ha recibido esta corriente, principalmente, relacionada con el marxismo, ha sabido reconstruir un aparato teórico conceptual de análisis sobre la realidad social, esto se debe a varios factores, entre ellos, su desapego con el marxismo leninismo, generando otras formas de analizar la realidad histórica social:

\footnotetext{
[...] el fin del marxismo oficial de la URSS liberó a Marx de la identificación pública con el leninismo en teoría y con los regímenes
} 


\begin{abstract}
leninistas en la práctica. Quedó muy claro que todavía había muchas y buenas razones para tener en cuenta lo que Marx tenía que decir acerca del mundo. Sobre todo porque, y está es la segunda razón, el mundo capitalista globalizado que surgió en la década de 1990 era en aspectos cruciales asombrosamente parecido al mundo anticipado por Marx en el Manifiesto comunista (Panfichi, 2011, p. 15).
\end{abstract}

Es importante señalar, que en el Bicentenario del nacimiento de $\mathrm{K}$. Marx, las condiciones sociopolíticas que este vislumbraba, se han transformado y los conflictos que actualmente se viven por xenofobía, racismo, discriminación étnica, sexual y de género, se han inmiscuido en una sociedad poscapitalista.

El papel de la Teoría Social, debe de asumirse de forma crítica, que no precisamente tenga que caer en un constructo ideológico, tratando de comprender sus partes como diferentes y/o a la vez relacionadas en forma de totalidad histórica, inherente al objeto de estudio de investigación.

Finalmente, en la actualidad ante la crisis científica, la Teoría Critica podría establecerse como parte de una revolución de paradigmas y de una forma en el que se pueden encaminar las Ciencias Sociales para crear autonomía. La desarticulación del lenguaje positivista en las Ciencias Sociales, podría ser el comienzo de un camino para la consolidación de un proyecto epistemológico crítico interdisciplinario, trascendiendo las fronteras epistemológicas institucionalizadas.

\section{Referencias}

[1] Adorno, Theodor (2008). Dialéctica Negativa La Jerga De La Autenticidad. Madrid: AKAL Básica De Bolsillo.

[2] Althusser, L. (1988). Ideología y aparatos ideológicos del Estado. Freud y Lacan. Buenos Aires: Nueva Visión.

[3] Barahona, Esther (2006). "Categorías y modelos en la Dialéctica negativa de Th. W. Adorno: critica al pensamiento idéntico". LOGOS. Anales del Seminario de Metafísica 39, 2006: 203-233.

[4] Bradner, Johann (1999) Sozialarbeit und Philosophie der Praxis in "Forum Kritische Psychologie", no. 40, traducción del alemán al español: Sieglin, Veronika, Trabajo Social y Filosofía de la Praxis. P- 1-9.

[5] Deleuze, Gilles, "Cuatro lecciones sobre Kant dictadas entre marzo y abril de 1978". Disponible en: <http://minhateca.com.br/rfbiblioteca/Biblioteca/DELEUZE*2c+Gilles/*2
$3 * 23 * 23+$ Deleuze $* 2 c+$ Guilles+-

+Cuatro+lecciones+sobre+Kant,302006694.pdf>[Consulta: 30 de mayo de 2015].

[6] Dussel, Enrique, Tema: Proximidad Curso Temas Selectos de Ética. Filosofía de la Liberación. Repositorio de la Facultad de Filosofía y Letras. UNAM, Colegio de Filosofía, Secretaría Académica, Facultad de Filosofía y Letras. Disponible en 〈http://ru.ffyl.unam.mx $>$ [Consulta: viernes 13 de marzo de 2015].

[7] Galindo, Jorge (2003). "Los retos de la interdisciplinariedad: Jürguen Habermas, entre sociología y filosofía" en Teoría Sociológica Contemporánea: Un debate inconcluso, compilado por Adriana García Andrade. México: Universidad Autónoma Metropolitana Unidad Azcapotzalco.

[8] Horkheimer, Max (2003). Teoría crítica. Buenos Aires: Amorrortu editores.

[9] Jay, Martin (1989). La imaginación dialéctica Una historia de la Escuela de Frankfurth. Madrid: Taurus.

[10] Laurent, Paul (1991). La escuela de francfort. México: Publicaciones Cruz O.S.A.

[11] Paez, Laura (2009). Teorías críticas de la modernidad ensayos y textos. México: UNAM-FES Acatlán.

[12] Panfichi, A. (2010). "Una entrevista con Eric Hobsbawm (1992)" en A contracorriente, Una revista de historia social y de literatura en América Latina. No. 3: p.p. 361-373.

[13] Salgado, Yulieth (2002). "Reseña de Abrir las Ciencias Sociales de Inmanuel Wallerstein". Revista de Ciencias Administrativas y Sociales 19 (enero-junio): 163-164.

[14] Sampaio, Miriam (2009). "La Teoría Crítica de la Escuela de Frankfurt, de la primera a la tercera generación: un recorrido histórico-sistemático". RIFP 34: 193-211.

[15] Tello, Felipe (2011). "Las esferas del reconocimiento en la teoría de Axel Honneth" en Revista Sociológica 26. p.p. 45-57. 